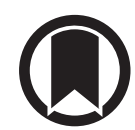

CrossMark

\title{
Asthma, COPD and overlap syndrome: a longitudinal study in young European adults
}

\author{
Roberto de Marco ${ }^{1}$, Alessandro Marcon ${ }^{1}$, Andrea Rossi ${ }^{2}$, Josep M. Antó $3,4,5,6$, \\ Isa Cerveri ${ }^{7}$, Thorarinn Gislason ${ }^{8}$, Joachim Heinrich $^{9,10}$, Christer Janson ${ }^{11}$, \\ Deborah Jarvis $^{12}$, Nino Kuenzli ${ }^{13,14}$, Bénédicte Leynaert ${ }^{15}$, Nicole Probst-Hensch ${ }^{13,14}$, \\ Cecilie Svanes $^{16,17}$, Matthias Wjst ${ }^{18,19}$ and Peter Burney ${ }^{12}$
}

Affiliations: 'Unit of Epidemiology and Medical Statistics, Department of Public Health and Community Medicine, University of Verona, Verona, Italy. ${ }^{2}$ Pulmonary Unit, Azienda Ospedaliera Universitaria Integrata and University of Verona, Verona, Italy. ${ }^{3}$ Centre for Research in Environmental Epidemiology (CREAL), Barcelona, Spain. ${ }^{4} \mathrm{Hospital}$ del Mar Medical Research Institute, Barcelona, Spain. ${ }^{5}$ Universitat Pompeu Fabra, Barcelona, Spain. ${ }^{6} \mathrm{ClBER}$ Epidemiología y Salud Pública (CIBERESP), Barcelona, Spain. ${ }^{7}$ Istituto di Ricovero e Cura a Carattere Scientifico San Matteo Hospital Foundation, University of Pavia, Pavia, Italy. ${ }^{8}$ Department of Respiratory Medicine and Sleep, Landspitali University Hospital and Faculty of Medicine, University of Iceland, Reykjavik, Iceland. ${ }^{9}$ Institute of Epidemiology I, Helmholtz Zentrum München, German Research Center for Environmental Health, Neuherberg, Munich, Germany. ${ }^{10}$ Institute and Outpatient Clinic for Occupational, Social and Environmental Medicine, Inner City Clinic, University Hospital of Munich, Ludwig-Maximilians University (LMU), Munich, Germany. ${ }^{11}$ Department of Medical Sciences: Respiratory Medicine and Allergology, Uppsala University Hospital, Uppsala, Sweden. ${ }^{12}$ Respiratory Epidemiology and Public Health Group, National Heart and Lung Institute, Imperial College, London, UK. ${ }^{13}$ Department of Epidemiology and Public Health, Swiss Tropical and Public Health Institute, Basel, Switzerland. ${ }^{14}$ University of Basel, Basel, Switzerland. ${ }^{15}$ InsermU1152-Epidemiology, Institut National de la Santé et de la Recherche Médicale, Faculté Paris Diderot, Paris, France. ${ }^{16}$ Bergen Respiratory Research Group, Centre for International Health, University of Bergen, Bergen, Norway. ${ }^{17}$ Department of Occupational Medicine, Haukeland University Hospital, Bergen, Norway. ${ }^{18}$ Comprehensive Pneumology Center, Institute of Lung Biology and Disease, Helmholtz Zentrum Muenchen, German Research Center for Environmental Health, Munich, Germany. ${ }^{19}$ Institute of Medical Statistics and Epidemiology, Technische Universitaet Muenchen, Munich, Germany.

Correspondence: Roberto de Marco, Unit of Epidemiology and Medical Statistics, Department of Public Health and Community Medicine, University of Verona, c/o Istituti Biologici II, Strada Le Grazie 8, 37134 Verona, Italy. E-mail: roberto.demarcodunivr.it

ABSTRACT We compared risk factors and clinical characteristics, 9-year lung function change and hospitalisation risk across subjects with the asthma-chronic obstructive pulmonary disease (COPD) overlap syndrome (ACOS), asthma or COPD alone, or none of these diseases.

Participants in the European Community Respiratory Health Survey in 1991-1993 (aged 20-44 years) and 1999-2001 were included. Chronic airflow obstruction was defined as pre-bronchodilator forced expiratory volume in $1 \mathrm{~s}$ (FEV1)/forced vital capacity<lower limit of normal on both occasions. Based on their history of respiratory symptoms, spirometry and risk factors, subjects were classified as having asthma alone ( $n=941)$, COPD alone $(n=166)$, ACOS $(n=218)$ and none of these $(n=5659)$.

Subjects with ACOS shared risk factors and clinical characteristics with subjects with asthma alone, but they had an earlier age of asthma onset. FEV1 change in the ACOS group $\left(-25.9 \mathrm{~mL} \cdot \mathrm{year}^{-1}\right)$ was similar to that in the asthma group $\left(-25.3 \mathrm{~mL} \cdot \mathrm{year}^{-1}\right)$, and lower $(\mathrm{p}<0.001)$ than in the COPD group $\left(-37.3 \mathrm{~mL} \cdot\right.$ year $\left.^{-1}\right)$. ACOS was associated with the highest hospitalisation rate.

Among young adults aged 20-44 years, ACOS seems to represent a form of severe asthma, characterised by more frequent hospitalisations, and to be the result of early-onset asthma that has progressed to fixed airflow obstruction.

@ERSpublications

The asthma-COPD overlap syndrome in young adults is a form of early-onset severe asthma with recurrent exacerbations http://ow.ly/MHkss 


\section{Introduction}

Asthma and chronic obstructive pulmonary disease (COPD) are a major public health problem, and they co-exist in a large proportion of subjects [1-6]. Patients with the asthma-COPD overlap syndrome (ACOS) have a more rapid disease progression, more respiratory symptoms, exacerbations, comorbidities and healthcare utilisation, compared to subjects with either disease alone [7-10].

According to some authors, ACOS is a syndrome in which older adults, generally with a significant history of smoking, have a partially reversible or fixed airflow obstruction and evidence of atopy or asthma [11]. It is still an open question whether ACOS is the result of asthma that has progressed to fixed airflow obstruction, or the expression of COPD in patients with airway hyperresponsiveness (AHR) or a specific disease entity $[12,13]$.

Few epidemiological studies have investigated the joint epidemiological distribution of asthma and COPD in the general population, as well as the long-term outcomes of ACOS [5, 11, 14]. Indeed, ACOS is often an exclusion criterion in studies investigating asthma or COPD $[9,15]$.

The aims of this prospective study were to assess, in an international cohort of young adults from the general population participating in the European Community Respiratory Health Survey (ECRHS), whether clinical characteristics and risk factors, long-term lung function decline and risk of being admitted to hospital or emergency room (ER) vary among subjects with asthma, COPD and ACOS.

\section{Methods}

Study design

The ECRHS I was an international multicentre study performed between 1991 and 1993 on random samples of young adults (aged 20-44 years) from the general population (www.ecrhs.org) [16]. From those who responded to a screening questionnaire (stage 1), a $20 \%$ "random sample" and an additional "symptomatic sample" (subjects with recent asthma-like symptoms or use of asthma medication) were selected for a clinical examination (stage 2; "baseline" examination). All participants in stage 2 were invited to take part in the "follow-up" examination (ECRHS II) between 1999 and 2002 [17]. Ethical approval was obtained for each centre from institutional or regional ethics committees and written consent was obtained from the participants.

The maximum pre-bronchodilator forced expiratory volume in $1 \mathrm{~s}(\mathrm{FEV} 1)$ and forced vital capacity (FVC) were measured at both surveys [18]. Chronic airflow obstruction was defined as a pre-bronchodilator FEV1/FVC<lower limit of normal (LLN) both at baseline and at follow-up [19]. Transient airflow obstruction was defined as a $\mathrm{FEV} 1 / \mathrm{FVC}<\mathrm{LLN}$ at baseline but not at follow-up. Predicted lung function values were computed [20]. AHR was defined as a decrease of $20 \%$ in FEV1 after a cumulative methacholine dose $\leqslant 1 \mathrm{mg}$. Allergen sensitisation was present if levels of serum immunoglobulin $\mathrm{E}$ for house dust mite, cat dander, Timothy grass or Cladosporium species were $>0.35 \mathrm{kU} \cdot \mathrm{L}^{-1}$. Body height and weight were measured and body mass index (BMI) was computed $\left(\mathrm{kg} \cdot \mathrm{m}^{-2}\right)$.

\section{Definitions}

At baseline, a subject was considered to have current asthma if 1) she/he reported to have or have had asthma AND one of asthma-like symptoms (wheezing/whistling in the chest, chest tightness, shortness of breath at rest/following strenuous activity/at night-time or asthma attacks); use of inhaled/oral medicines for breathing problems in the last year; AHR; or transient airflow obstruction; or 2) she/he reported asthma-like symptoms in the last year AND had AHR.

Since post-bronchodilator spirometry was not performed in ECRHS I and II, a subject was considered to have COPD at baseline if she/he had pre-bronchodilator chronic airflow obstruction AND either 1) symptoms (shortness of breath after strenuous activity, dyspnoea (trouble with breathing) or chronic bronchitis (having cough or phlegm on most days for as long as 3 months each year for $\geqslant 2$ years)); or 2) a history of active smoking ( $\geqslant 10$ pack-years) [21], or occupational exposure to vapours, dust, gas or fumes (indicated by a positive answer to "Have you ever worked in a job which exposed you to vapours, gas, dust or fumes?").

Received: Jan 192015 | Accepted after revision: April 222015 | First published online: June 252015

Support statement: Supported by the European Commission, as part of their Quality of Life programme. See the online supplementary material for sources of funding for the local European Community Respiratory Health Survey studies included in this article. The funding organisations had no role in the design of the study; the collection, analysis, and interpretation of the data; and the decision to approve publication of the finished manuscript. Funding information for this article has been deposited with FundRef.

This article has supplementary material available from erj.ersjournals.com 
The validity of our definition of pre-bronchodilator chronic airflow obstruction was assessed using preliminary post-bronchodilator lung function data collected in ECRHS III (2010-2014). The findings (online supplementary material) suggest that "chronic airflow obstruction" is a valid measurement of fixed airflow obstruction (sensitivity $80.2 \%$ and specificity $98.3 \%$ ), and that the rate of misclassification is very similar for subjects with and without asthma (positive predictive value $73.9 \%$ and $69.3 \%$, respectively).

Finally, subjects were classified into four mutually exclusive groups at baseline [5]: 1) "healthy" subjects (neither current asthma nor COPD); 2) current asthma alone (asthma without COPD); 3) ACOS (both current asthma and COPD); and 4) COPD alone (COPD without asthma).

A similar classification was applied at follow-up to evaluate whether disease status was stable over the study period (online supplementary material).

The longitudinal outcomes were as follows. 1) Absolute FEV1 (and FVC) change over follow-up ((valuefollow-up - valuebaseline)/follow-up time) $\left(\mathrm{mL} \cdot \mathrm{year}^{-1}\right)$ and FEV1 (and FVC) change as a percentage of baseline value $(100 \times($ valuefollow-up - valuebaseline)/(valuebaseline $\times$ follow-up time)) (percentage per year). Negative values represent decline. 2) Risk (\%) of hospitalisations and/or ER visits because of breathing problems over follow-up.

\section{Statistical analysis}

Prevalence of risk factors and clinical characteristics at baseline (as defined in tables 2 and 3 and the online supplementary material) were adjusted for sex and age (continuous variable) by logistic regression. Multiple linear and logistic regression models were fitted to data using lung function change and hospitalisation rates, respectively, as dependent variables, and disease status as the main independent variable. Adjustment variables were age, height and BMI (treated as continuous variables), sex, education, occupational exposure at baseline, lifetime smoking exposure and BMI change over time. All models included a random intercept for ECRHS centres and type of sample (random versus symptomatic) [22]. Missing values were deleted listwise. The statistical analyses were performed using Stata 13.1 (StataCorp, College Station, TX, USA).

In sensitivity analyses, 1) asthma was defined as a positive answer to "have you ever had asthma?" (self-reported asthma) at baseline and COPD was defined as the presence of chronic airflow obstruction (disregarding the presence of symptoms and exposures); and 2) ECRHS centres were set as a random intercept and type of sample was set as a fixed effect.

\section{Results}

18356 subjects from 29 centres in 14 countries participated in the ECRHS I stage 2 (1991-1993 baseline), of whom 15716 (86\%) were from the random sample (fig. 1). Overall, 10933 (60\%) subjects attended the second survey, of whom 9175 (84\%) were from the random sample. Subjects participating in ECRHS II were older, less likely to have ever smoked and had better lung function at baseline than subjects who did not (online supplementary table S4). Mean \pm SD follow-up time was $9 \pm 1$ years (range $4-12$ years). Among participants in the second survey who had data on lung function and asthma, 5659 healthy subjects, 941 subjects with current asthma alone, 218 subjects with ACOS and 166 subjects with COPD alone were identified, while 131 subjects could not be classified (fig. 1). The distribution of subjects across the different sub-definitions of current asthma and COPD is reported in the online supplementary material (table S5).

Among participants in ECRHS II, the characteristics of the subjects included and not included in the analyses were similar, with the exception that the latter were slightly younger and more likely to have AHR (table 1). Disease status was relatively stable over time (online supplementary table S3): the percentage of subjects that were classified, both at baseline and at follow-up, in the same disease group ranged between 75.7\% (COPD alone) and 93.0\% (healthy subjects).

\section{Baseline characteristics and risk factors}

Subjects with current asthma alone were younger (mean \pm SD $33.6 \pm 7.2$ years) and more likely to be women than subjects in the other groups (table 2), while subjects with COPD were the oldest (36.0 \pm 6.5 years). Smoking was more frequent among subjects with ACOS or COPD. Among lifetime smokers, the prevalence of heavy smoking ( $\geqslant 15$ pack-years) was $51.5 \%$ for subjects with COPD alone (median (interquartile range) 16.8 (15.9) pack-years), and it ranged from 27.1\% (healthy 9.8 (13.8) pack-years) to $35.1 \%$ (ACOS 10.3 (20.1) pack-years) in the other groups $(\mathrm{p}<0.001)$. Occupational exposures were reported more frequently in the COPD (57.4\%) and asthma (45.9\%) groups than in the reference category $(42.0 \%)$ ( $p=0.001)$. Family asthma and childhood respiratory infections were the most frequent in subjects with asthma or ACOS $(\mathrm{p}<0.001)$. 


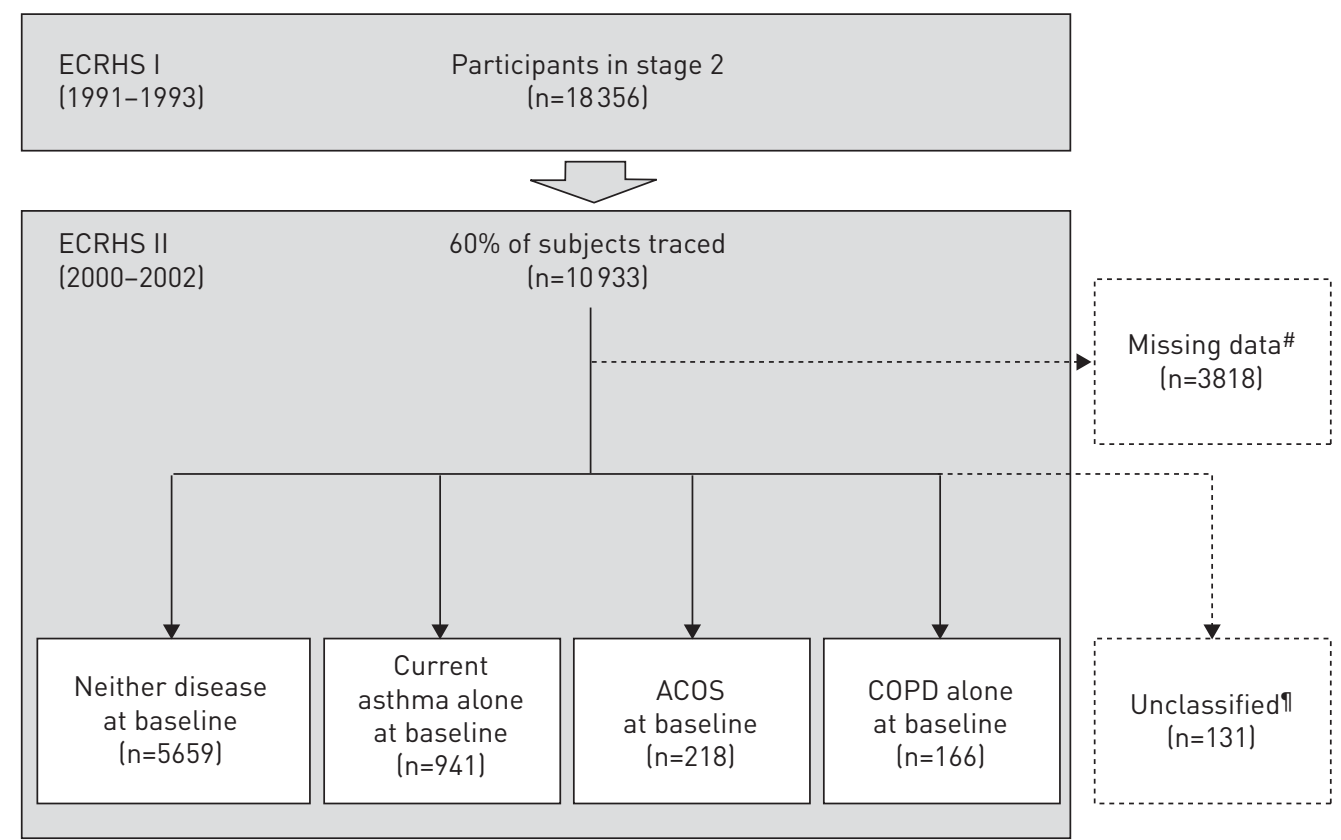

FIGURE 1 Number of subjects included in the analyses. ECRHS: European Community Respiratory Health Survey; ACOS: asthma-chronic obstructive pulmonary disease (COPD) overlap syndrome. \#: for 3810 subjects spirometry was not available either at baseline and/or follow-up, and eight subjects did not answer the question on asthma at baseline; " ${ }^{\text {: }}$ subjects who reported asthma at baseline but did not fulfil the criteria for current asthma $(n=67)$, and subjects who had chronic airflow obstruction but did not fulfil the criteria for COPD ( $n=64)$, were not considered in the main analysis.

Information on asthma onset was available for 705 (74.9\%) subjects with asthma alone and 170 (78.0\%) with ACOS. On average, subjects with ACOS had an earlier age at asthma onset (14.9 versus 17.2 years, $\mathrm{p}=0.016$ ), a longer disease duration (27.7 versus 24.8 years, $\mathrm{p}=0.003$ ), a greater percentage of inhaled corticosteroid use $(30.2$ versus $20.6 \%, \mathrm{p}=0.003)$ and more frequent asthma attacks in the last year $(11.2$ versus $6.2, \mathrm{p}=0.034)$.

The prevalence rates of wheezing, dyspnoea, chronic bronchitis, allergic rhinitis, eczema and allergen sensitisation, as well as the use of medicines and hospital/ER admissions, were the highest for subjects with ACOS or current asthma alone (all p<0.001) (table 3). The prevalence of AHR ranged from 3.5\% (healthy) to $92.1 \%$ (ACOS) $(\mathrm{p}<0.001$ ). Subjects with $\mathrm{FEV} 1<80 \%$ predicted were $4.5 \%$ for current asthma alone, $16.4 \%$ for COPD alone and $33.1 \%$ for the ACOS group $(\mathrm{p}<0.001)$.

TABLE 1 Baseline (European Community Respiratory Health Survey (ECRHS) I) characteristics of the subjects participating in ECRHS II

\begin{tabular}{lccc} 
& $\begin{array}{c}\text { Subjects included } \\
\text { in the analyses }\end{array}$ & $\begin{array}{c}\text { Subjects not included } \\
\text { in the analyses }\end{array}$ & p-value \\
\hline Subjects & 6984 & 3949 & 0.93 \\
Female & $3708(53)$ & $2100(53)$ & $<0.001$ \\
Age years & $34.3 \pm 7.1$ & $33.7 \pm 7.2$ & 0.53 \\
BMI kg:m - $^{\text {Low education }}$ & $24.1 \pm 4.0$ & $24.1 \pm 4.0$ & 0.83 \\
Smoking habits & $875(13)$ & $499(13)$ & 0.16 \\
$\quad$ Nonsmoker & $3067(45)$ & $1793(46)$ & \\
$\quad<15$ pack-years & $2499(36)$ & $1347(35)$ & 0.49 \\
Sen pack-years & $1321(19)$ & $722(19)$ & 0.95 \\
FEV $\%$ predicted & $2130(34)$ & $970(35)$ & 0.001 \\
AHR & $105.0 \pm 13.5$ & $398(18)$ &
\end{tabular}

Data are presented as $n, n(\%)$ or mean $\pm S D$, unless otherwise stated. BMI: body mass index; FEV1: forced expiratory volume in $1 \mathrm{~s}$; AHR: airway hyperresponsiveness. 
TABLE 2 Sex- and age-adjusted prevalence of sociodemographic characteristics, environmental exposures and risk factors at baseline

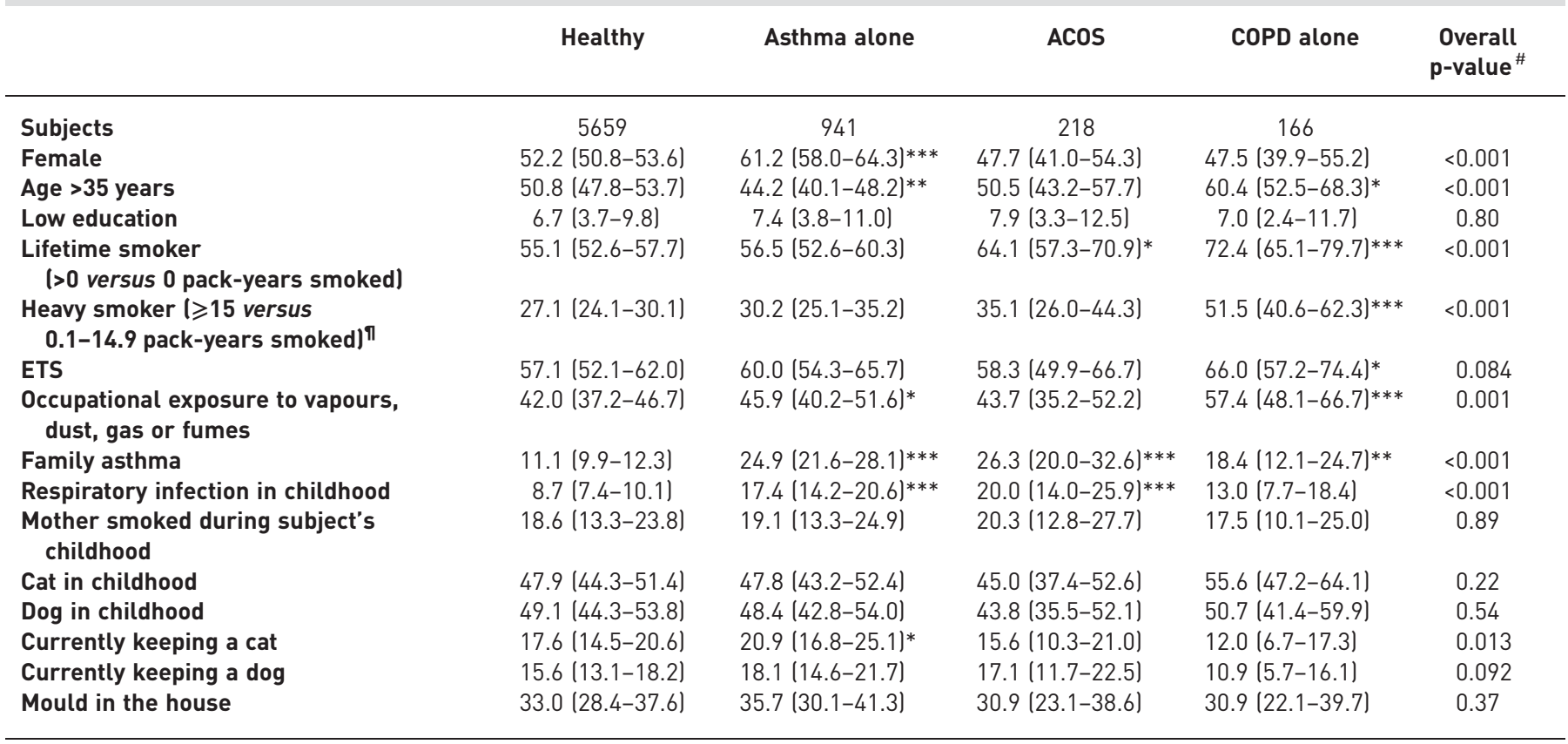

Data are presented as $\mathrm{n}$ or $\%(95 \% \mathrm{Cl})$, unless otherwise stated. Data were adjusted using logistic regression models with the characteristic in the first column as the dependent variable, sex, age (with the exception of sex and age, which were only adjusted for age and sex, respectively), and disease status as independent variables, and a random intercept term for European Community Respiratory Health Survey centre and sample. ACOS: asthma-chronic obstructive pulmonary disease (COPD) overlap syndrome; ETS: environmental tobacco smoke. ${ }^{\#}$ : refers to the comparison across the groups; $p<0.05$ indicates that at least one of the prevalence rates is significantly different from the others; ๆ: lifetime nonsmokers excluded. ${ }^{* *}: p<0.01{ }^{* * *}: p<0.001$ for the comparison of the disease group with the healthy category.

Change in lung function and risk of hospitalisation

Mean change in FEV1 and hospitalisation rates at follow-up are reported in table 4. After adjusting for potential confounders (table 5), subjects with COPD alone had a $-7.64(95 \% \mathrm{CI}-12.6--2.66) \mathrm{mL} \cdot \mathrm{year}^{-1}$ greater change in FEV1 compared to healthy subjects $(\mathrm{p}=0.007)$, whereas their FVC change was -13.83 $(95 \%$ CI $-19.96--7.70) \mathrm{mL} \cdot y e \mathrm{r}^{-1}$ greater $(<0.001)$. Lung function change was similar in asthma, ACOS and healthy subjects. Similar results were obtained when analysing change in lung function as a percentage of baseline value. Subjects with COPD alone, asthma alone and ACOS had a two-fold, four-fold, and five-fold greater risk of reporting hospital/ER admissions over the follow-up, respectively, with respect to the reference group $(\mathrm{p}=0.080)$.

\section{Sensitivity analyses}

When the disease groups were identified according to alternative definitions of asthma (self-reported asthma at baseline) and COPD (chronic obstruction), the distribution of risk factors and clinical characteristics were similar (online supplementary tables S6 and S7), with the exceptions that the difference in smoking exposure between subjects with ACOS and healthy subjects, as well as the difference in occupational exposures between subjects with COPD and healthy subjects, shifted to the null. The results of the analyses on lung function change and hospitalisation risk were fully confirmed both using these alternative definitions (table S8), and using an alternative hierarchical model structure (data not shown).

\section{Discussion}

The aim of this paper was to better understand ACOS by investigating its similarities and differences with respect to asthma and COPD alone. This is one of the first studies investigating ACOS in an international population-based cohort. We studied young adults in an age range when disease evolution is still only minimally masked by the effects of cumulative exposure to risk factors and comorbidities [23]. We found that subjects with both asthma and COPD shared with asthmatic subjects risk factors and clinical characteristics, and had a 9-year lung function decline similar to subjects with asthma, significantly lower than in COPD. 
TABLE 3 Sex- and age-adjusted prevalence of clinical characteristics at baseline

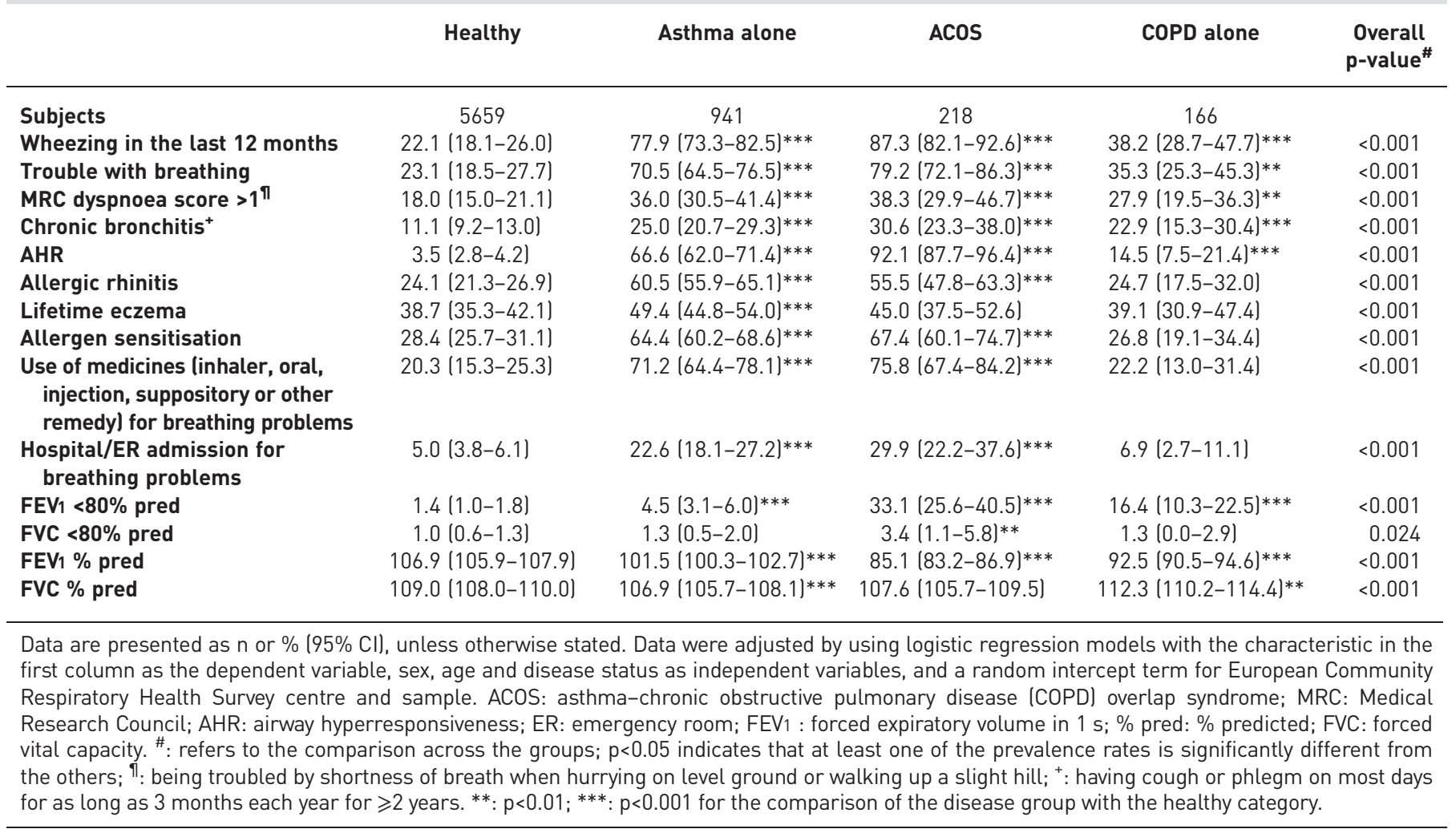

\section{Clinical characteristics and risk factors}

Previous reports showing that subjects with ACOS have a pattern of risk factors that is intermediate between asthma and COPD, but more exacerbations and greater severity than subjects with either disease alone $[7,24]$, raised the hypothesis that ACOS could be a specific disease entity $[12,13,25]$.

As far as we know, studies on ACOS were usually cross-sectional and based on selected groups of elderly patients or medical record data and collected limited clinical information [8-10]. It is therefore not surprising that our findings are only in partial agreement with previous evidence, as they point out that subjects with ACOS have the same clinical profile and risk factors as asthmatics (even if they represent a

TABLE 4 Change of lung function and prevalence of hospital/emergency room (ER) admissions for breathing problems over follow-up

\begin{tabular}{|c|c|c|c|c|c|}
\hline & Healthy & Asthma alone & AcOS & COPD alone & $\begin{array}{l}\text { Overall } \\
\text { p-value }\end{array}$ \\
\hline Subjects & 5659 & 941 & 218 & 166 & \\
\hline FEV 1 change $\%$ of baseline $\cdot y e^{-1}$ & $-0.69(-0.83--0.55)$ & $-0.68(-0.83--0.53)$ & $-0.66(-0.84--0.47)$ & $-1.17(-1.37--0.97)^{* * *}$ & $<0.001$ \\
\hline FVC change $\mathrm{mL} \cdot$ year $^{-1}$ & $-19.8(-25.6--14.1)$ & $-21.3(-27.4--15.2)$ & $-25.5(-33.0--18.0)^{*}$ & $-37.0(-45.0--29.0)^{* * *}$ & $<0.001$ \\
\hline FVC change $\%$ of baseline year $^{-1}$ & $-0.42(-0.55--0.29)$ & $-0.45(-0.59--0.31)$ & $-0.55(-0.72--0.38)^{*}$ & $-0.81(-0.99--0.63)^{* * *}$ & $<0.001$ \\
\hline
\end{tabular}

Data are presented as $\mathrm{n}$ or mean (95\% Cl), unless otherwise stated. Change of lung function was adjusted using linear regression models, with forced expiratory volume in $1 \mathrm{~s}\left(\mathrm{FEV}_{1}\right)$ or forced vital capacity (FVC) change as dependent variables, sex, age, height and disease status as independent variables, and a random intercept term for European Community Respiratory Health Survey (ECRHS) centre and sample. A negative value represents lung function decline. Prevalence of hospital admissions for breathing problems was adjusted using a logistic regression model, with hospital/ER admissions as dependent variables, sex, age and disease status as independent variables, and a random intercept term for ECRHS centre and sample. ACOS: asthma-chronic obstructive pulmonary disease (COPD) overlap syndrome; AHR: airway hyperresponsiveness. "\#: refers to the comparison across the groups; " : present if a subject answered positively to one or both of "Since the last survey, have you spent a night in hospital?" or "Have you visited a hospital casualty department or ER because of breathing problems?" ${ }^{*}: p<0.05 ;{ }^{* *}: p<0.01 ;{ }^{* * *}: p<0.001$ for the comparison of the disease group with the "healthy" category. 
TABLE 5 Change of lung function and risk of hospital/emergency room (ER) admission for breathing problems over follow-up, for subjects with current asthma, chronic obstructive pulmonary disease (COPD) or both, compared with healthy subjects

\begin{tabular}{|c|c|c|c|c|}
\hline & Asthma alone & ACOS & COPD alone & $\begin{array}{c}\text { p-value } \\
\text { heterogeneity }\end{array}$ \\
\hline FEV1 change $\mathrm{mL} \cdot$ year $^{-1}$ & $0.69(-1.65-3.02)$ & $1.62(-2.76-5.99)$ & $-7.64(-12.6--2.66)^{* *}$ & 0.007 \\
\hline FVC change $\mathrm{mL} \cdot$ year $^{-1}$ & $-0.92(-3.79-1.96)$ & $-4.84(-10.23-0.54)$ & $-13.83(-19.96--7.70)^{* * *}$ & $<0.001$ \\
\hline FVC change $\%$ of baseline year $^{-1}$ & $-0.01(-0.08-0.05)$ & $-0.11(-0.23-0.01)$ & $-0.30(-0.43--0.16)^{* * *}$ & $<0.001$ \\
\hline $\begin{array}{l}\text { Hospital/ER admission for breathing } \\
\text { problems }{ }^{\pi} \text { OR }(95 \% \text { CI) with respect } \\
\text { to the healthy category }\end{array}$ & $3.76(2.84-4.99) * * *$ & $5.12(3.24-8.10)^{* * *}$ & $2.10(1.06-4.13)^{*}$ & 0.080 \\
\hline
\end{tabular}

Data are presented as mean difference $(95 \% \mathrm{Cl})$ with respect to the healthy category, unless otherwise stated. Regression coefficients were obtained using linear regression models, with forced expiratory volume in $1 \mathrm{~s}$ (FEV 1 ) or forced vital capacity (FVC) change as dependent variables, sex, age, height, body mass index (BMI), education level, occupational exposure to vapours, gas, dust or fumes, disease status at baseline or lifetime smoking exposure (five-level variable coded as follows. 0: lifetime nonsmoker; 1: past smoker <15 pack-years; 2: past smoker $\geqslant 15$ pack-years; 3 : current smoker $<15$ pack-years; or 4: current smoker $\geqslant 15$ pack-years) and change in BMI over follow-up as independent variables, and a random intercept term for European Community Respiratory Health Survey (ECHRS) centre and sample. A negative value represents a greater decline with respect to the healthy reference category. Odds ratios were obtained using a logistic regression model, with hospital/emergency room (ER) admissions as dependent variables, sex, age, BMI, education level, occupational exposure to vapours, gas, dust or fumes, disease status at baseline, lifetime smoking exposure and change in BMI over follow-up as independent variables, and a random intercept term for ECRHS centre and sample. ACOS: asthma-COPD overlap syndrome. \#: p-value for the heterogeneity of the association across the groups, obtained by testing the difference across regression coefficients using Wald test; $p<0.05$ indicates that at least one of the regression coefficients is significantly different from the others, i.e. that the change in lung function (or risk of hospital/ER admissions) with respect to the reference healthy category differs for at least one disease group compared to the others; ${ }^{\text {I }}$ : present if a subject answered positively to one or both of "Since the last survey, have you spent a night in hospital?" or "Have you visited a hospital casualty department or ER because of breathing problems?" *: $p<0.05 ;^{* *}: p<0.01 ;{ }^{* * *}: p<0.001$ for the comparison of the disease group with the healthy category.

more severe subgroup), which was quite different from that of subjects with COPD. Indeed, they shared the same prevalence of allergen sensitisation, allergic rhinitis and eczema with asthmatics. Almost all of them (92.1\%) had AHR. Furthermore, they had the same increased prevalence of family asthma history and childhood respiratory infections [26]. Like asthmatics, subjects with ACOS had a greater prevalence of respiratory symptoms, use of medicines and hospital/ER admissions than young COPD patients. With respect to other asthmatics, subjects with ACOS had an earlier onset and a longer duration of asthma, a higher frequency of smoking and a higher prevalence of male sex.

In agreement with previous studies on the general population [27], and in contrast with clinical studies on older patients [9], we found that subjects with ACOS had worse lung function at baseline than subjects with asthma or COPD alone. With respect to other asthmatics, this result may reflect airway remodelling or a failure to attain maximal airway growth because of an early disease onset [23], or both. Indeed, a correlation between disease duration and airway remodelling has been reported [28]. The poorer lung function with respect to COPD is probably due to the fact that the effect of the exposures that lead to COPD can only be recognised at older ages [29].

In the main analyses, definitions of active asthma and COPD were adopted by using information on current symptoms or exposures. For this reason, the distribution of some symptoms and risk factors may have depended on the definitions used. However, when we adopted alternative definitions of asthma and COPD that did not consider the presence of symptoms and risk factors, the distribution of characteristics across disease groups was very similar, with a few exceptions regarding smoking and occupational exposures. In a joint statement of the Global Initiative for Asthma and the Global Initiative fror Chronic Obstructive Lung Disease [2], ACOS is defined as "persistent airflow limitation with several features usually associated with asthma and several features usually associated with COPD". Accordingly, ACOS was defined as the overlap between asthma and COPD in our study. Thus, it is worth noting that different distributions of characteristics in ACOS versus asthma (or ACOS versus COPD) did not depend on the definitions used, but they reflected peculiarities of ACOS compared to asthma or COPD alone.

\section{Change in lung function and risk of hospitalisation}

While COPD is characterised by an accelerated, although variable FEV1 decline [21], many asthmatic patients experience a slow decline [30]. Accordingly, our findings document that subjects with asthma alone had a 9-year decline in lung function that was not different from that of people without respiratory diseases. Subjects with ACOS, who had the poorest FEV1 and FVC at baseline, had a similar FEV1 and FVC decline to subjects with asthma, but a significantly lower decline than in COPD. One explanation is 
that the mechanisms and pathways of airflow obstruction in ACOS and COPD may be different. In the ACOS group, they may be due to acquired deficits in lung growth very early in life [26], while in COPD they are mainly due to a steeper decline in adult life because of risk factor exposure. The younger age at asthma onset in subjects with ACOS in our study is consistent with this hypothesis. The very high prevalence of AHR in ACOS also fits this picture. In fact, AHR is associated with COPD and worse lung function, even when AHR is measured extremely early in life [31,32]. Alternatively, subjects with ACOS may have had a severe insult or more frequent exacerbations early in life, which may have caused a significant impairment in lung function that is no longer progressive. However, this might be at least in part due to the efficacy of treatments in preventing lung function deterioration in asthma [33]. To our knowledge, there are only two other longitudinal studies comparing lung function decline of subjects with ACOS, asthma and COPD $[8,34]$. Both are clinical studies on older patients and used different disease definitions compared to our study, and the samples investigated were smaller and not representative of the vast majority of cases in the general population. Results from the most recent of these two studies support our conclusion [34], while the other found that lung function decline in ACOS patients was more similar to that of COPD patients, and greater than in asthma patients [8].

In agreement with previous clinical studies [10, 26, 35], our population study showed that ACOS subjects, who had the lowest FEV1 \% pred at baseline, had a rate of hospital/ER admissions for breathing problems during the follow-up that was more than double that of subjects with either disease alone. Indeed, asthma with fixed airflow obstruction is one of the main clinical phenotypes of uncontrolled severe asthma [36], characterised by a poor prognosis and recurrent exacerbations, and reduced FEV1 is an important risk factor for multiple exacerbations both in asthma and COPD [37].

\section{Study limitations}

As in many other large-scale surveys started in the 1990s, post-bronchodilator spirometry was not available. As a consequence, some asthmatic subjects with fully reversible obstruction could have been falsely classified as COPD. To minimise this bias, we used chronic airflow obstruction (pre-bronchodilator FEV1/FVC $<$ LLN in both studies, 9 years apart) as a spirometric criterion of COPD. A pilot evaluation, based on preliminary post-bronchodilator lung function data of ECRHS III (2010-2014), supports the validity of pre-bronchodilator chronic airflow obstruction as an indicator of fixed obstruction. In fact, sensitivity and specificity were $76.7 \%$ and $98.8 \%$, respectively, in subjects without asthma, and $86.4 \%$ and $94.3 \%$, respectively, in subjects with asthma (online supplementary table S1). This supports the fact that our definition of chronic airflow obstruction captured the majority of subjects with "true" post-bronchodilator obstruction, and that it excluded virtually all subjects without. Moreover, high and fairly similar positive predictive values for subjects with (73.9\%) and without (69.3\%) asthma suggest non-differential misclassification, strengthening the validity of comparisons between subjects with ACOS and subjects with COPD alone.

Either respiratory symptoms and/or active smoking/occupational exposures were necessary, in combination with the spirometrical criterion, to define COPD [2, 21]. This resulted in a more specific definition compared to the definition based on spirometry alone [38]. Longitudinal studies with post-bronchodilator lung function data will be needed to adequately compare the level and severity of airflow obstruction in subjects with ACOS and COPD.

Since the aim of the study was not to estimate disease prevalence in the population but to compare characteristics across disease groups, this analysis included both a random subsample of respondents and all the subjects who reported symptoms suggestive of asthma at the screening questionnaire. However, as a consequence of the random sampling of the population participating at ECRHS stage 1, the subjects from the disease groups investigated are representative of the disease in the population.

Unfortunately, information on inflammatory markers to characterise the ACOS phenotypes was not available in our study [13]. Finally, the participation rate was not particularly high. However, the comparison of baseline information between subjects who did and did not participate showed that the two groups were similar.

\section{Conclusion}

Our findings suggest that, at least among young adults aged 20-44 years, ACOS represents a form of severe asthma, characterised by more frequent exacerbations, and it is likely to be the result of early asthma that has progressed to fixed airflow obstruction, possibly because of airway remodelling.

\section{References}

1 Bahadori K, Doyle-Waters MM, Marra C, et al. Economic burden of asthma: a systematic review. BMC Pulm Med 2009; 9: 24 .

2 Global Initiative for Chronic Obstructive Lung Disease. Global Strategy for the Diagnosis, Management, and Prevention of Chronic Obstructive Pulmonary Disease. www.goldcopd.org/guidelines-global-strategy-for-diagnosismanagement.html/ Date last accessed: April 8, 2015. Date last updated: January 2015. 
3 de Marco R, Cappa V, Accordini S, et al. Trends in the prevalence of asthma and allergic rhinitis in Italy between 1991 and 2010. Eur Respir J 2012; 39: 883-892.

4 Gershon A, Guan J, Victor JC, et al. Quantifying health service use for chronic obstructive pulmonary disease. Am J Respir Crit Care Med 2013; 187: 596-601.

5 de Marco R, Pesce G, Marcon A, et al. The coexistence of asthma and chronic obstructive pulmonary disease (COPD): prevalence and risk factors in young, middle-aged and elderly people from the general population. PLoS One 2013; 8: e62985.

6 Hersh CP, Jacobson FL, Gill R, et al. Computed tomography phenotypes in severe, early-onset chronic obstructive pulmonary disease. COPD 2007; 4: 331-337.

7 Louie S, Zeki AA, Schivo M, et al. The asthma-chronic obstructive pulmonary disease overlap syndrome: pharmacotherapeutic considerations. Expert Rev Clin Pharmacol 2013; 6: 197-219.

8 Contoli M, Baraldo S, Marku B, et al. Fixed airflow obstruction due to asthma or chronic obstructive pulmonary disease: 5-year follow-up. J Allergy Clin Immunol 2010; 125: 830-837.

9 Hardin M, Silverman EK, Barr RG, et al. The clinical features of the overlap between COPD and asthma. Respir Res 2011; 12: 127.

10 Kauppi P, Kupiainen H, Lindqvist A, et al. Overlap syndrome of asthma and COPD predicts low quality of life. J Asthma 2011; 48: 279-285.

11 Soriano JB, Davis KJ, Coleman B, et al. The proportional Venn diagram of obstructive lung disease: two approximations from the United States and the United Kingdom. Chest 2003; 124: 474-481.

12 Zeki AA, Schivo M, Chan A, et al. The asthma-COPD overlap syndrome: a common clinical problem in the elderly. J Allergy (Cairo) 2011; 2011: 861926.

13 Al-Kassimi FA, Alhamad EH. A challenge to the seven widely believed concepts of COPD. Int J Chron Obstruct Pulmon Dis 2013; 8: 21-30.

14 Viegi G, Matteelli G, Angino A, et al. The proportional Venn diagram of obstructive lung disease in the Italian general population. Chest 2004; 126: 1093-1101.

15 de Marco R, Accordini S, Cerveri I, et al. Incidence of chronic obstructive pulmonary disease in a cohort of young adults according to the presence of chronic cough and phlegm. Am J Respir Crit Care Med 2007; 175: 32-39.

16 Burney PG, Luczynska C, Chinn S, et al. The European Community Respiratory Health Survey. Eur Respir J 1994; 7: 954-960.

17 European Community Respiratory Health Survey II Steering Committee. The European Community Respiratory Health Survey II. Eur Respir J 2002; 20: 1071-1079.

18 American Thoracic Society. Standardization of spirometry, 1994 update. Am J Respir Crit Care Med 1995; 152: $1107-1136$.

19 Cerveri I, Corsico AG, Accordini S, et al. Underestimation of airflow obstruction among young adults using FEV $1 /$ FVC $<70 \%$ as a fixed cut-off: a longitudinal evaluation of clinical and functional outcomes. Thorax 2008; 63: $1040-1045$.

20 Quanjer PH, Tammeling GJ, Cotes JE, et al. Lung volumes and forced ventilatory flows. Report Working Party Standardization of Lung Function Tests, European Community for Steel and Coal. Official Statement of the European Respiratory Society. Eur Respir J 1993; 6: Suppl. 16, 5-40.

21 Vestbo J, Edwards LD, Scanlon PD, et al. Changes in forced expiratory volume in 1 second over time in COPD. N Engl J Med 2011; 365: 1184-1192.

22 Marcon A, Corsico A, Cazzoletti L, et al. Body mass index, weight gain, and other determinants of lung function decline in adult asthma. J Allergy Clin Immunol 2009; 123: 1069-1074.

23 Burney P. Variable loss of lung function in COPD. N Engl J Med 2011; 365: 1246-1247.

24 Gibson PG, Simpson JL. The overlap syndrome of asthma and COPD: what are its features and how important is it? Thorax 2009; 64: 728-735.

25 Piras B, Miravitlles M. The overlap phenotype: the (missing) link between asthma and COPD. Multidiscip Respir Med 2012; 7: 8.

26 Svanes C, Sunyer J, Plana E, et al. Early life origins of chronic obstructive pulmonary disease. Thorax 2010; 65: 14-20.

27 Mannino DM, Gagnon RC, Petty TL, et al. Obstructive lung disease and low lung function in adults in the United States: data from the National Health and Nutrition Examination Survey, 1988-1994. Arch Intern Med 2000; 160: 1683-1689.

28 Brown PJ, Greville HW, Finucane KE. Asthma and irreversible airflow obstruction. Thorax 1984; 39: 131-136.

29 de Marco R, Accordini S, Marcon A, et al. Risk factors for chronic obstructive pulmonary disease in a European cohort of young adults. Am J Respir Crit Care Med 2011; 183: 891-897.

30 Panettieri RA Jr, Covar R, Grant E, et al. Natural history of asthma: persistence versus progression - does the beginning predict the end? J Allergy Clin Immunol 2008; 121: 607-613.

31 Bisgaard H, Jensen SM, Bønnelykke K. Interaction between asthma and lung function growth in early life. Am J Respir Crit Care Med 2012; 185: 1183-1189.

32 Marcon A, Cerveri I, Wjst M, et al. Can an airway challenge test predict respiratory diseases? A population-based international study. J Allergy Clin Immunol 2014; 133: 104-110.

33 de Marco R, Marcon A, Jarvis D, et al. Inhaled steroids are associated with reduced lung function decline in subjects with asthma with elevated total IgE. J Allergy Clin Immunol 2007; 119: 611-617.

$34 \mathrm{Fu}$ JJ, Gibson PG, Simpson JL, et al. Longitudinal changes in clinical outcomes in older patients with asthma, COPD and asthma-COPD overlap syndrome. Respiration 2014; 87: 63-74.

35 Andersén H, Lampela P, Nevanlinna A, et al. High hospital burden in overlap syndrome of asthma and COPD. Clin Respir J 2013; 7: 342-346.

36 Campo P, Rodríguez F, Sánchez-García $S$, et al. Phenotypes and endotypes of uncontrolled severe asthma: new treatments. J Investig Allergol Clin Immunol 2013; 23: 76-88.

37 Hurst JR, Vestbo J, Anzueto A, et al. Susceptibility to exacerbation in chronic obstructive pulmonary disease. N Engl J Med 2010; 363: 1128-1138.

38 de Marco R, Accordini S, Antò JM, et al. Long-term outcomes in mild/moderate chronic obstructive pulmonary disease in the European Community Respiratory Health Survey. Am J Respir Crit Care Med 2009; 180: 956-963. 